\title{
Predictive Modeling of Particle-Laden,
}

Quarterly Progress Report No. 1

covering the period of September 1 to December 1, 1992

\author{
to the Department of Energy \\ Pittsburgh Energy Technology Center \\ Pittsburgh. PA
}
Project Manager: Frank Shaffer
)

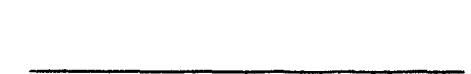
from the Department of Chemical Engineering
Carnegie Mellon University
Pittsburgh, PA 15213

Ciaduate Students: E.J. Bolio and C.M. Hrenya

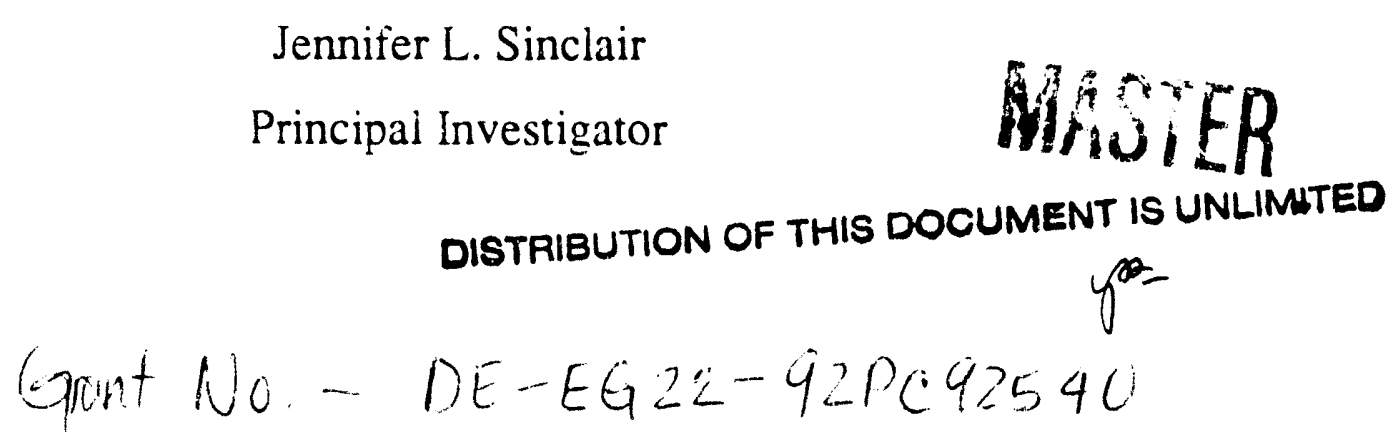




\section{TABLE OF CONTENTS}

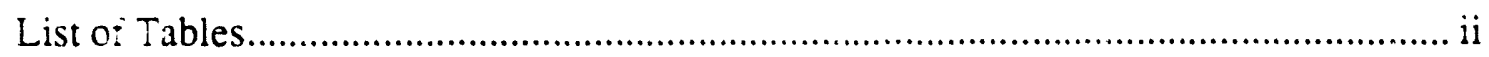

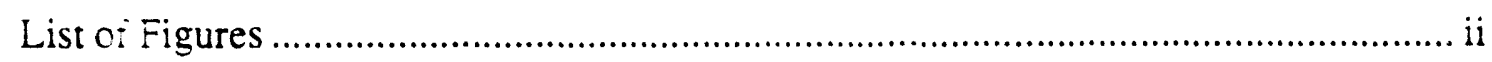

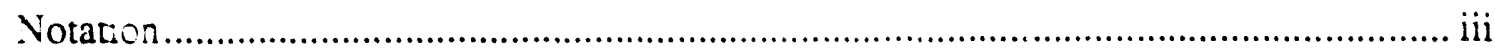

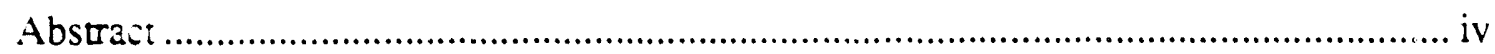

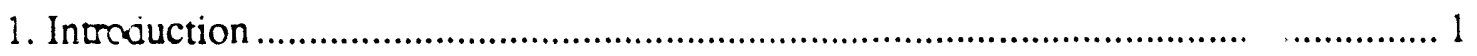

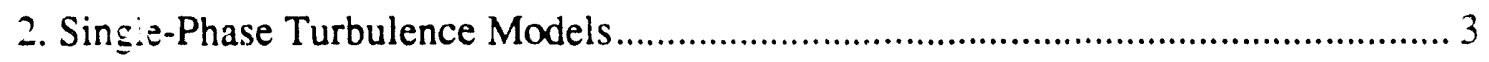

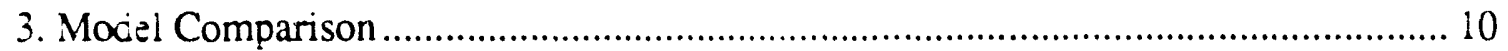

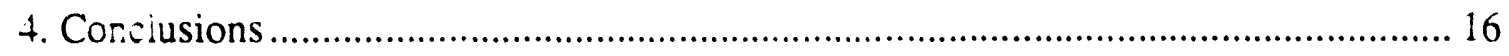

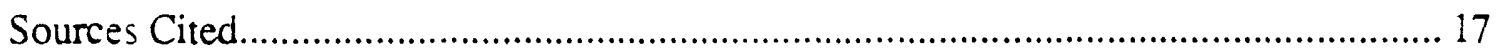




\section{List of Tables}

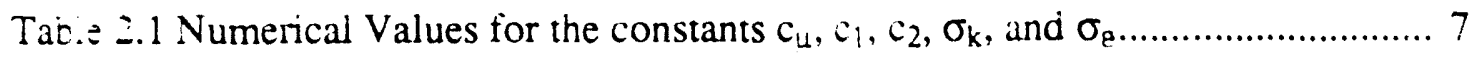

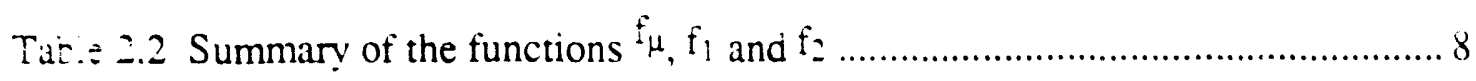

Tat.: 2.3 Summary of the terms $D$ and $E$, and boundary conditions for $k$ and $\varepsilon \ldots \ldots . . .9$

Tar.: 3.1 Physical parameters for the experimental system of Schildknecht et al.

$119^{-2}$

\section{List of Figures}

Figures 3.1 a, 3.1b Comparison of the model predictions for the mean velocity wits :-2e experimental data of Schildknecht et al. (1979)...

Fig $=2$ s 3.2a. 3.2b Comparison of the model predictions for the turbulent kinetic ene: $:-$ with the experimental data of Schildknecht et al. (1979).

Fig - 3.3a, 3.3b Model predictions for the eddy viscosity for the system of

Sch: iknecht et al. (1979).

Figu- 3.4 Model predictions for the turbulent kinetic energy dissipation rate in the $\because$ ail region for the system of Schildknecht et al. (1979). 


\title{
Notation
}

\author{
Variables: \\ $c_{\mu}, c_{.}:_{2} \quad$ constants in $\mathrm{k}-\varepsilon$ model \\ D term included in the $k$ equation \\ E term included in the $\varepsilon$ equation \\ $f_{\mu}, f_{i}, i_{2}$ functions in $k-\varepsilon$ model \\ $\mathrm{k} \quad$ gas fluctuating kinetic energy \\ P pressure \\ $r \quad$ radial coordinate \\ $\mathrm{R} \quad$ pipe radius \\ $\operatorname{Re} \quad$ Reynolds number based on the centerline velocity \\ $\mathrm{R}_{\mathrm{T}} \quad$ turbulent Reynolds number \\ $R_{y} \quad$ turbulent Reynolds number based on $y$ \\ $: \quad$ time \\ $U_{\tau} \quad$ frictional velocity \\ $\mathrm{V} \quad$ fluid mean velocity \\ $\mathrm{V}_{\mathrm{CL}} \quad$ centerline fluid velocity \\ $v^{\prime} \quad$ fluid fluctuating velocity \\ $\mathrm{x}$ spatial coordinate \\ $y \quad$ spatial coordinate normal to the wail \\ $y^{+} \quad$ dimensionless spatial coordinate
}

\section{Greek symbols:}

$\begin{array}{ll}\varepsilon & \text { fluid fluctuating kinetic energy dissipation rate } \\ \lambda & \text { Taylor's length scale } \\ \downarrow & \text { fluid intrinsic viscosity } \\ \nu & \text { fluid kinematic viscosity } \\ v_{T} & \text { eddy viscosity } \\ \rho & \text { fluid density } \\ \sigma_{k} & \text { Prandtl turbulent number for } k \\ \sigma_{\varepsilon} & \text { Prandtl turbulent number for } \varepsilon\end{array}$

The :-ascript next indicates the property value at the point next to the wall. 


\begin{abstract}
The : accessful prediction of particle-laden. turbulent flows relies heavily on the representation of turbulence in the gas phase. Several types of turbulence models for sing:2-phase gas flow have been developed which compare reasonably well with exper:mental data. In the present work, a "low-Reynolds" $k-\varepsilon$ closure model is chosen to descr.ve the Reynolds stresses associated with gas-phase turbulence. This closure scheme, which involves transport equations for the turbulent kinetic energy and its dissization rate, is valid in the turbulent core as well as the viscous sublayer. Several versions of the low-Reynolds $k-\varepsilon$ closure are documented in the literature. However, even :aose models which are similar in theory often differ considerably in their quanatative and qualitative predictions, making the selection of such a model a difficult task.
\end{abstract}

The z:urpose of this progress report is to document our findings on the performance of ten diffe:ent versions of the low-Reynolds $k-\varepsilon$ model on predicting fully developed pipe flow. The predictions are compared with the experimental data of Schildknecht, et al. $\left(19^{-0}:\right.$

Wit: :ne exception of the model put forth by Hoffman (1975), the predictions of all the closures show reasonable agreement for the mean velocity profile. However, important quar:atative differences exist for the turbulent kinetic energy profile. In addition, the precicied eddy viscosity profile and the wall-region profile of the turbulent kinetic energy diss:zation rate exhibit both quantitative and qualitative differences. An effort to extend the resent comparisons to inciude experimental measurements of other researchers is recommended in order to further evaluate the performance of the models. 


\section{Introduction}

Two-zase, particle-laden, turbulent flows occur in a variety of engineering applications. Exampies include the processes of fluid catalytic cracking, coal gasification, calcination of alumina, desulfurization of flue gases, and combustion of a wide variety of fuels to environmental standards (Rhodes and Geldart. 1989). These flows cannot be described simpiy due to the complex interaction of the gas and solid velocity fields. The radial nonuniformity of voidage, high gas-solid slip velocities, and the downflow of solids in the riser annulus in dense-phase conveying, are all types of flow phenomena resulting from this interaction.

Previous modeling efforts have been able to qualitatively predict the flow phenomena mentioned above by treating the particle-particle and particle-wall collisions analogous to molecuiar collisions as described by the kinetic theory of gases (Sinclair and Jackson, 1989: Pita and Sundaresan, 1991). However, a quantitative comparison of these model predictions with experimental two-phase data would have been premature since these modeis did not incorporate gas-phase turbulenie. Louge et al. (1991) proposed the first two-pnase model which included a description of gas-phase turbulence. This model employs a high-Reynolds one-equation turbulence closure model and a kinetic-theory based approach which is valid only at the limit of very dilute flows with elastic particleparticie collisions. Although their predictions were in agreement with reported expermental measurements, they also concluded that a better turbulence closure is required to improve the model performance.

The overall goal of this research is to expand on the previous efforts in order to develop a modei which accurately predicts the behavior of gas-solid mixtures in fully developed 
isothermal pipe flow. This effort begins by introducing a more general gas-phase turbulence closure scheme, namely a low-Reynolds version of the $k-\varepsilon$ closure model. Several versions of this model have been documented in the literature. Although these versions are similar in form, they have been developed using different approaches and therefore are expected to predict differently. In a review of the existing low-Reynolds $k-\varepsilon$ models, Patel et al. (1985) confirmed that important differences do exist between the predictions of these models. Hence, the selection of one of these versions to serve as the basis for the two-phase model becomes a non-trivial task. Unfortunately, Patel's review did not compare the model predictions with experimental data obtained from pipe flow. In addition, since the publication of this review, more versions of the $k-\varepsilon$ model have been published. Thus, the immediate focus of this research is to evaluate the ability of all existing low-Reynolds $k-\varepsilon$ closure models to predict fully developed pipe flow. 


\section{Single-Phase Turbulence Models}

The description of turbulent flows for a single phase is still very much an active research area. The different approaches to the problem are divided into those models that solve the Navier-Stokes (NS) equations in an exact form, and those models that solved the time-averaged NS equations, i.e., the Reynolds equations. Currently, the exact solution of the IS equations is only possible for relatively simple problems due to the high computational cost of resolving the small scale of the turbulent phenomena. Thus, those models based of the Reynolds equations offer the most viable choice from the engineering standpoint.

For a pressure-gradient driven flow, the Reynolds equations are:

$$
\left.\rho \frac{\partial V_{i}}{\partial t}+\rho V_{j} \frac{\partial V_{i}}{\partial x_{j}}=-\frac{\partial P}{\partial x_{i}}+\frac{\partial}{\partial x_{j}} \mid \mu \frac{\partial V_{i}}{\partial x_{j}}-\rho \overline{v^{\prime}{ }_{i} v_{j}^{\prime}}\right)
$$

where $V:$ and $P$ are the mean velocity and pressure which result from the time-averaging process.

The term $\rho \overline{v^{\prime}{ }_{i}{ }^{\prime} j}$, which is also introduced by the averaging process, represents the transport of momentum due to the fluctuating turbulent motion. It is the determination of this correlation of the fluctuating velocities which is the central problem of all the turbulence models based on the Reynolds equations. Several alternatives are available to approximate this correlation, ranging from the simplest model based on Prandtl's mixing length to the rather sophisticated Reynolds stress models which utilize one transport equation for every correlation $\overline{v^{\prime} v^{\prime} j}$. Among these choices, a two-equation closure model. the $k-\varepsilon$ model, has proven to be the most widely tested and successfully applied 
turoulence model (Rodi, 1979). Recent studies (Martinuzzi and Pollard, 1989a, 1989b) indicate that the $k-\varepsilon$ model performs better than the conceptually superior Reynolds stress modiels.

The $\mathrm{k}-\varepsilon$ model is based on the concept of the eddy viscosity $v_{\mathrm{T}}$. An assumption of this concept is that the turbulent stresses, like the viscous stresses, are proportional to meanvelocity gradients:

$$
\overline{v^{\prime}{ }_{i} v_{j}^{\prime}}=-v_{T}\left(\underline{\underline{V}}+\underline{\underline{\nabla v^{T}}}\right)
$$

This analogy is further extended by assuming that the eddy viscosity is proportional to a veiocity scale and a length scale that characterizes the turbulent motion, just as the intrinsic viscosity is proportional to the average velocity and the mean free path of the moiecules. Kolmogorov and Prandtl independently proposed to :hoose the turbulent kinetic energy, $\mathrm{k}$, as the variable representing the velocity scale. For the length scale, severai variables such as the kinetic energy dissipation rate $\varepsilon$, the turbulent frequency, the turoulent vorticity, and others have been suggested. The choice of $\varepsilon$ as the length scale variable has proven the most successful (Rodi, 1979). Hence, $v_{-}$is prescribed via the Kolmogorov Prandtl expression:

$$
v_{T}=c_{\mu} \frac{k^{2}}{\varepsilon}
$$

The profiles for $k$ and $\varepsilon$ are obtained by solving two transport equations. For this reason, the $k-\varepsilon$ model is known as a two-equation closure scheme. The $k$ and the $\varepsilon$ equations are given in cylindrical coordinates for a fully developed flow at steady state:

$$
\frac{1}{\mathrm{r}} \frac{\partial}{\partial \mathrm{r}} \cdot \mathrm{r} \frac{v_{\mathrm{T}}}{\sigma_{\mathrm{k}}} \frac{\partial \mathrm{k}}{\partial \mathrm{r} !}+\nu_{\mathrm{T}}\left(\frac{\partial \mathrm{V}}{\partial \mathrm{r}}\right)^{2}-\varepsilon=0
$$




$$
\frac{1}{\mathrm{r}} \frac{\partial}{\partial \mathrm{r}}\left[\mathrm{r} \frac{\nu_{\mathrm{T}}}{\sigma_{\varepsilon}} \frac{\partial \varepsilon}{\partial \mathrm{r}}\right]+\frac{c_{1} \nu_{T} \varepsilon}{\mathrm{k}}\left(\frac{\partial \mathrm{V}}{\partial \mathrm{r}}\right)^{2}-\frac{c_{2} \varepsilon^{2}}{\mathrm{k}}=0
$$

The $\mathrm{k}$ transport equation describes the balance between the diffusive transport by turbulent motion (first term), the production by interaction of turbulent stresses and mean velocity gradients (second term), and the destruction by dissipation (third term). Similar processes are represented by the three terms of the $\varepsilon$ equation. $\sigma_{k}$ and $\sigma_{\varepsilon}$ are the empirically determined turbulent Prandtl numbers for $k$ and $\varepsilon$, respectively. $c_{\mu}, c_{1}$ and $c_{2}$ are also constants.

The particular form of the $k-\varepsilon$ model outlined above is the original one as first published by Jones and Launder (1972). This form is very successful in the prediction of singlephase flows with sufficiently high Reynolds number where viscous effects are negligible. Hence, this form of the model is not applicable near a solid boundary. For confined flows, wall functions are often applied at a small distance away from the wall, bridging over the viscous sublayer. These functions, which are based on experimental data, cannot easily be extended to two-phase flow. In order to avoid using wall functions, modifications to the original $k-\varepsilon$ model have been made. The modified forms of the $k-\varepsilon$ model, known as "low-Reynolds" $k-\varepsilon$ models, can be applied continuously throughout the turbulent core and the viscous sublayer. The modifications to the original model are:

1. The dissipation rate $\varepsilon$ is replaced by a "dissipation variable" $\varepsilon^{*}=\varepsilon-D$. Physically, $\varepsilon^{*}$ represents the isotropic part of the dissipation of the random kinetic energy. The use of the dissipation variable $\varepsilon^{*}$ generates an additional term $\mathrm{D}$ in the $\mathrm{k}$ equation. The term $\mathrm{D}$ is chosen in such a way so that the dissipation variable $\varepsilon^{*}$ can be equated to zero at the wall, a numerically convenient boundary condition. 
2. An extra term $\mathrm{E}$ is added to the $\varepsilon$ equation in order to yield realistic $\mathrm{k}$ and $\varepsilon$ distributions in the viscous sublayer.

3. Viscous diffusion of $k$ and $\varepsilon$ is accounted for by the addition of the intrinsic viscosity to the corresponding diffusion terms.

4. The functions $f_{\mu}, f_{1}$ and $f_{2}$ are introduced to modify the constants $c_{\mu}, c_{1}$ and $c_{2}$. These functions depend on different dimensionless numbers related to the turbulence phenomena, the velocity field. and the geometry:

$$
\begin{array}{lll}
\mathrm{R}_{\mathrm{T}}=\frac{\mathrm{k}^{2}}{\mathrm{v}} \quad(2.6) & \mathrm{R}_{\mathrm{y}}=\frac{\sqrt{\mathrm{k} y}}{\mathrm{v}}(2.7) & \mathrm{Re}_{\text {avg }}=\frac{2 \mathrm{R}\langle\mathrm{V}\rangle}{\mathrm{v}} \\
\mathrm{y}=\mathrm{R}-\mathrm{r}(2.9) & \mathrm{y}^{+}=\frac{\mathrm{U}_{\tau} \mathrm{y}}{\mathrm{v}}(2.10) & \lambda^{2}=\frac{10 v \mathrm{k}}{\varepsilon}
\end{array}
$$

5. The use of wall functions is eliminated, and boundary conditions for $k$ and $\varepsilon$ are enforced at the wall.

With these modifications included, the equations (2.3), (2.4) and (2.5) become:

$$
\begin{gathered}
\frac{1}{r} \frac{\partial}{\partial r}\left[r\left(v+\frac{v_{T}}{\sigma_{k}}\right) \frac{\partial k}{\partial r}\right]+v_{T}\left(\frac{\partial V}{\partial r}\right)^{2}-\varepsilon-D=0 \\
\frac{1}{r} \frac{\partial}{\partial r}\left[r\left(v+\frac{v_{T}}{\sigma_{\varepsilon}}\right) \frac{\partial \varepsilon}{\partial r}\right]+\frac{c_{1} f_{1} v_{T} \varepsilon}{k}\left(\frac{\partial V}{\partial r}\right)^{2}-\frac{c_{2} f_{2} \varepsilon^{2}}{k}+E=0 \\
v_{T}=c_{\mu} f_{\mu} \frac{k^{2}}{\varepsilon}
\end{gathered}
$$

Several single-phase "low-Reynolds" $k-\varepsilon$ models have been proposed and excellent reviews on this subject are offered by Patel et al. (1985) and Shih (1988). However, since the publication of these reviews, more versions have been published. All of the 
versions differ in the numerical values assigned to the constants. the expressions used for $D, E, f_{\mu}, f_{1}$ and $f_{2}$, as well as in the boundary conditions for $k$ and $\varepsilon$. Table 2.1 summarizes the numerical values assigned to the constants of ten versions and the original $\mathrm{k}-\varepsilon$ model. The models are listed in chronological order.

\begin{tabular}{|l|c|c|c|c|c|c|}
\hline \multicolumn{1}{|c|}{ Model } & Initials & $c_{\mu}$ & $c_{1}$ & $c_{2}$ & $\sigma_{k}$ & $\sigma_{\varepsilon}$ \\
\hline \hline Original k- $\varepsilon$ & $\mathrm{k}-\varepsilon$ & 0.09 & 1.45 & 2.0 & 1.0 & 1.3 \\
\hline Jones and Launder (1972) & $\mathrm{JL}$ & 0.09 & 1.45 & 2.0 & 1.0 & 1.3 \\
\hline Launder and Sharma (1975) & $\mathrm{LS}$ & 0.09 & 1.44 & 1.92 & 1.0 & 1.3 \\
\hline Hoffman (1975) & $\mathrm{HO}$ & 0.09 & 1.81 & 2.0 & 2.0 & 3.0 \\
\hline Hassid and Poreh (1978) & $\mathrm{HP}$ & 0.09 & 1.45 & 2.0 & 1.0 & 1.3 \\
\hline Dutoya and Michard (1981) & $\mathrm{DM}$ & 0 & 1.35 & 2.0 & 0.9 & 0.95 \\
\hline Chien (1982) & $\mathrm{CH}$ & 0.09 & 1.35 & 1.8 & 1.0 & 1.3 \\
\hline Lam and Bremhorst (1981) & LB & 0.09 & 1.44 & 1.92 & 1.0 & 1.3 \\
\hline Lai and So (1990) & LSO & 0.09 & 1.35 & 1.8 & 1.0 & 1.3 \\
\hline Mvong and Kasagi (1990) & MK & 0.09 & 1.4 & 1.8 & 1.4 & 1.3 \\
\hline So. Zhang and Speziale & SZS & 0.096 & 1.5 & 1.83 & 0.75 & 1.45 \\
\hline (1991) & & & & & & \\
\hline
\end{tabular}

Table 2.1 Numerical Values for the constants $c_{\mu}, c_{1}, c_{2}, \sigma_{k}$, and $\sigma_{\varepsilon}$

Table 2.2 shows the different functions $f_{\mu}, f_{1}$ and $f_{2}$ for the same group of models. Table 2.3 lists the terms $\mathrm{D}$ and $\mathrm{E}$, and the boundary conditions for $\mathrm{k}$ and $\varepsilon$.

1 In the definition of $R_{T}, v_{T}$ and $\lambda$, this model requires the substitution of $\varepsilon$ by $\varepsilon+D / p$ 


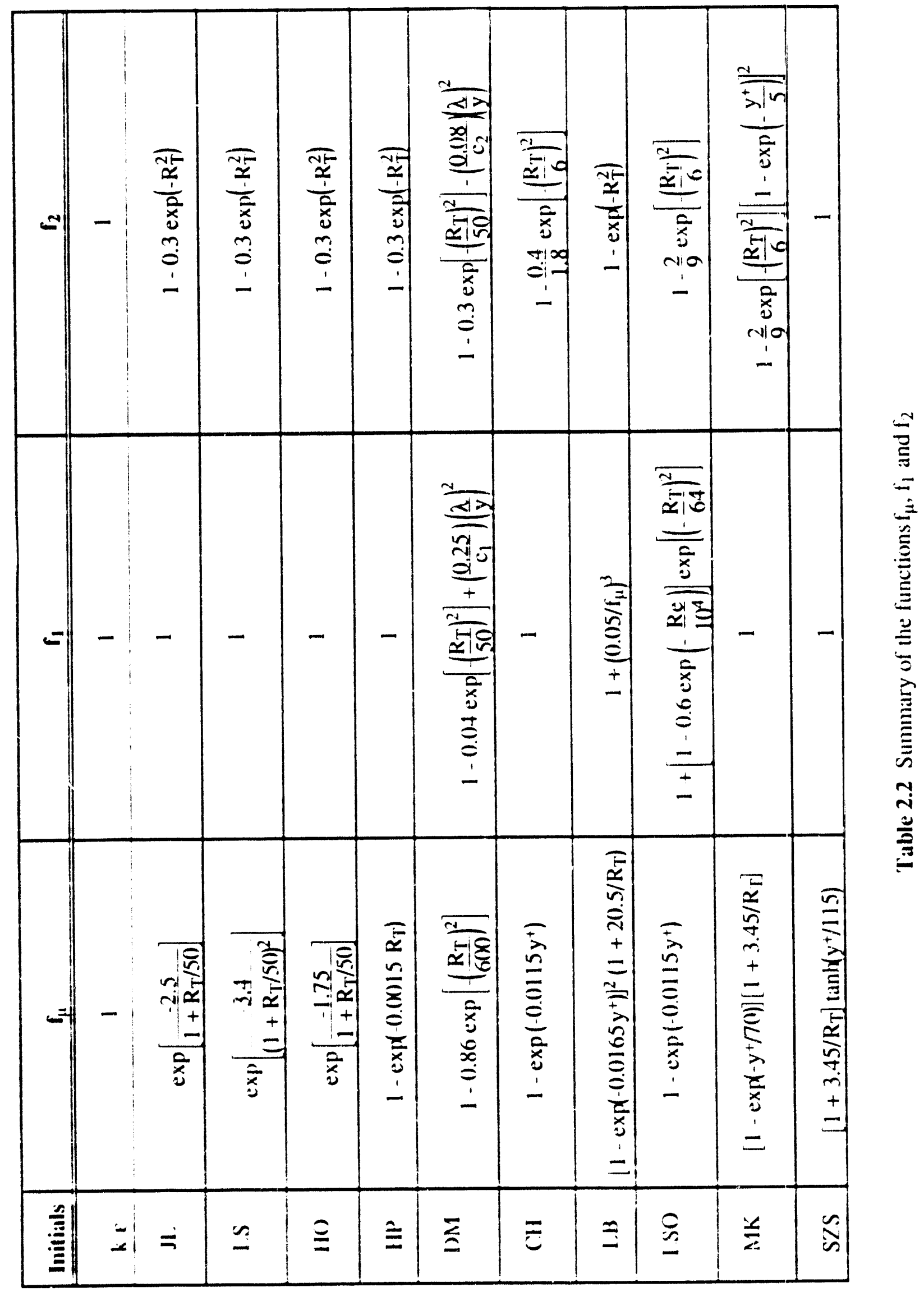




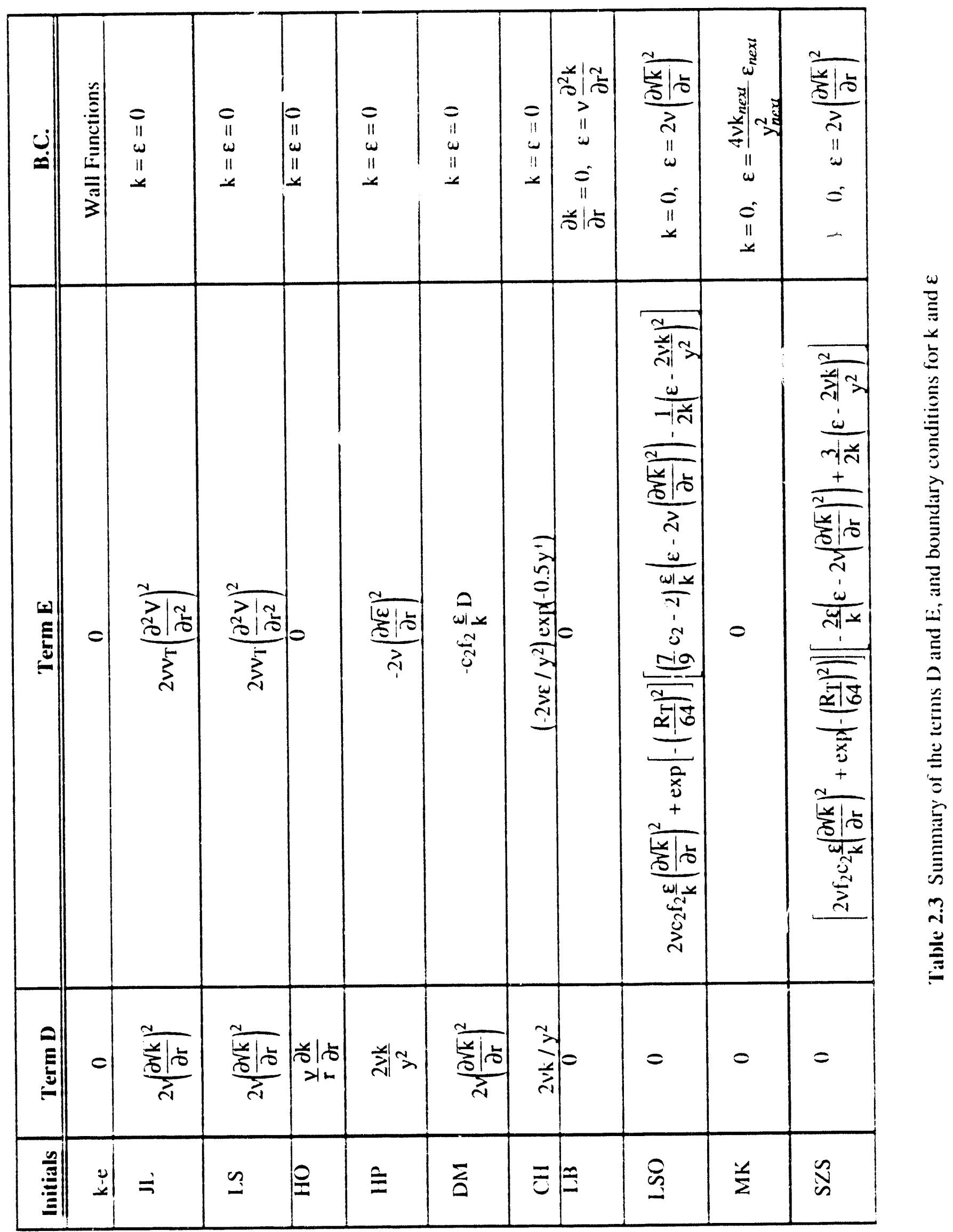




\section{Model Comparison}

in this section, comparisons are made between the predictions of the ten different versions 0 ? Schildknecht et al. (1979). The primary purpose of this comparison is to gauge the success of each of the different closures in an effort to determine which closure should be used as the base turbulence model for the two-phase flow description.

The data of Schildknecht et al. was obtained using a hot-wire probe to measure the veiocity profiles for the fully-developed flow of air in a cylincirical pipe. The Reynolds number based on the centerline velocity is 21,800 . Other details of the experiment are listed in Table 3.1.

\begin{tabular}{|l|c|}
\hline Pipe radius $[\mathrm{m}]$ & 0.025 \\
\hline Air density $[\mathrm{kg} / \mathrm{m} 3]$ & 1.204554 \\
\hline Air viscosity $[\mathrm{kg} / \mathrm{m}-\mathrm{s}]$ & 0.0000175 \\
\hline Centerline Velocity $\mathrm{V}_{\mathrm{CL}}[\mathrm{m} / \mathrm{s}]$ & 6.3438 \\
\hline Frictional Velocity $\mathrm{U}_{\tau}[\mathrm{m} / \mathrm{s}]$ & 0.29 \\
\hline
\end{tabular}

Table 3.1 Physical parameters for the experimental system of Schildknecht et al. (1979)

Figures $3.1 \mathrm{a}$ and $3.1 \mathrm{~b}$ show a comparison of the mean gas velocity prediction of the ten closures with the experimental data. In these plots, the frictional velocity matches the measured value. The predictions of the ten versions are divided in two plots for clarity, and the model predictions are organized chronologically. Figure 3.1 a shows the first five models, and Figure $3.1 \mathrm{~b}$ shows the five most recent models. 

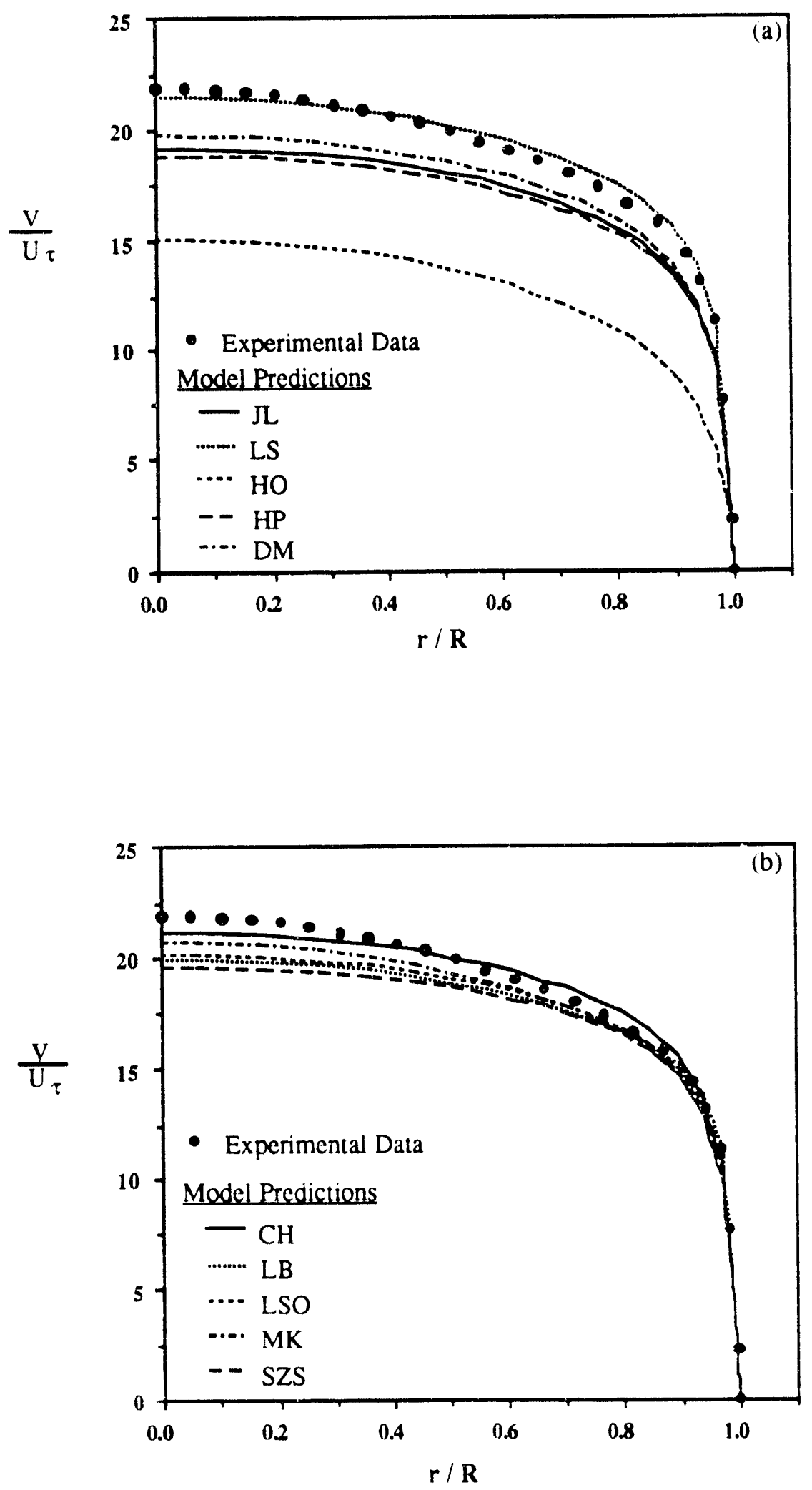

Figures 3.1a, 3.1b Comparison of the model predictions for the mean velocity with the expenmental data by Schildknecht et al (1979). The frictional velocity $U_{\tau}$ is $0.29 \mathrm{~m} / \mathrm{s}$. 
All of the models are in good agreement with the experimental measurements, within $15^{\circ}$ of the $V_{C L}$ experimental value, except for the model of Hoffman which has a $31.5 \%$ error. The reasonable agreements obtained for the mean velocity predictions should not be surprising since the prediction of this profile was the primary purpose of all of the models. The LS and $\mathrm{CH}$ models offer the best overall agreement, while the $\mathrm{HO}$ presents the worst overall agreement.

In Figure 3.2a and 3.2b, we show a comparison of the predicted turbulent kinetic energy $\mathrm{k}$ with the experimental data. Even though all of the predictions have the same quaiitative trend as the experimental data, significant variations in the quantitative predictions exist. With the exception of the MK model, all of the models overpredict the centerline turbulent kinetic energy, with differences as large as $77.5 \%$ (HP model). The predictions of the location of characteristic peak of these profiles are very good. However, the predicted magnitude of the peak differs from model to model. Three models, DM, LSO and SZS, ove1, rict the peak value for $\mathrm{k}$, and the largest overprediction has a difference of $9.2 \%$ (LSO model). The rest of the models underpredict the $\mathrm{k}$ peak with differences as large as $29.7 \%$ (LS model).

In F: gures 3.3a and 3.3b, we show the model predictions of the eddy viscosity. The agreement of all the models with respect to each other is poor. Differences in the predicted centerline values can vary more than $100 \%$. Furthermore, qualitative differences in the profiles are also noticed. While the majority of the profiles have a parabolic shape, the MK presents a change in concavity near the centerline, which is in agreement with the observed behavior. 

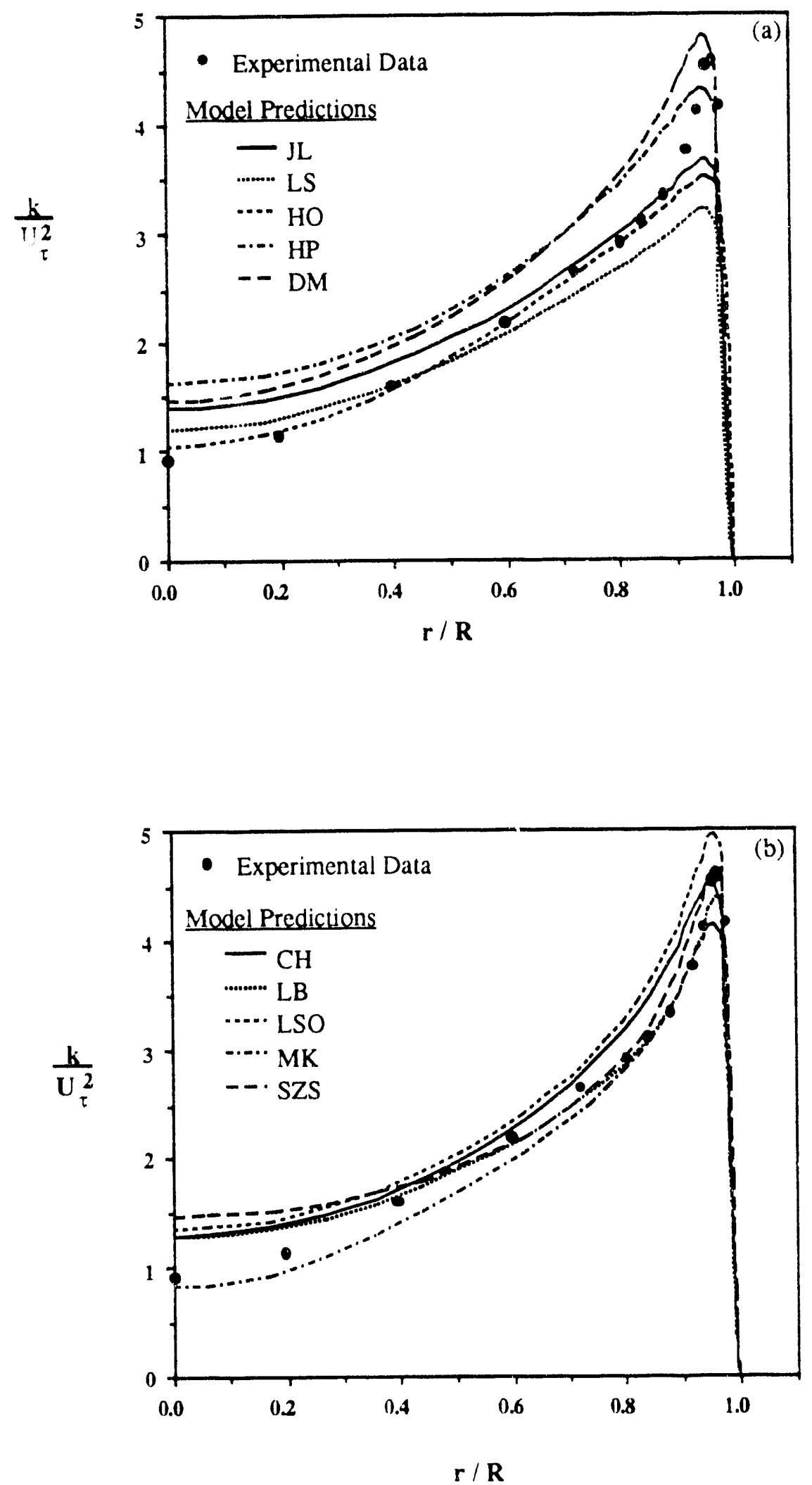

Figures 3.2a, 3.2b Comparison of the model predictions for the turbulent kinetic energy with the experimental data by Schildknecht et al (1979). The frictional velocity $U_{\tau}$ is 

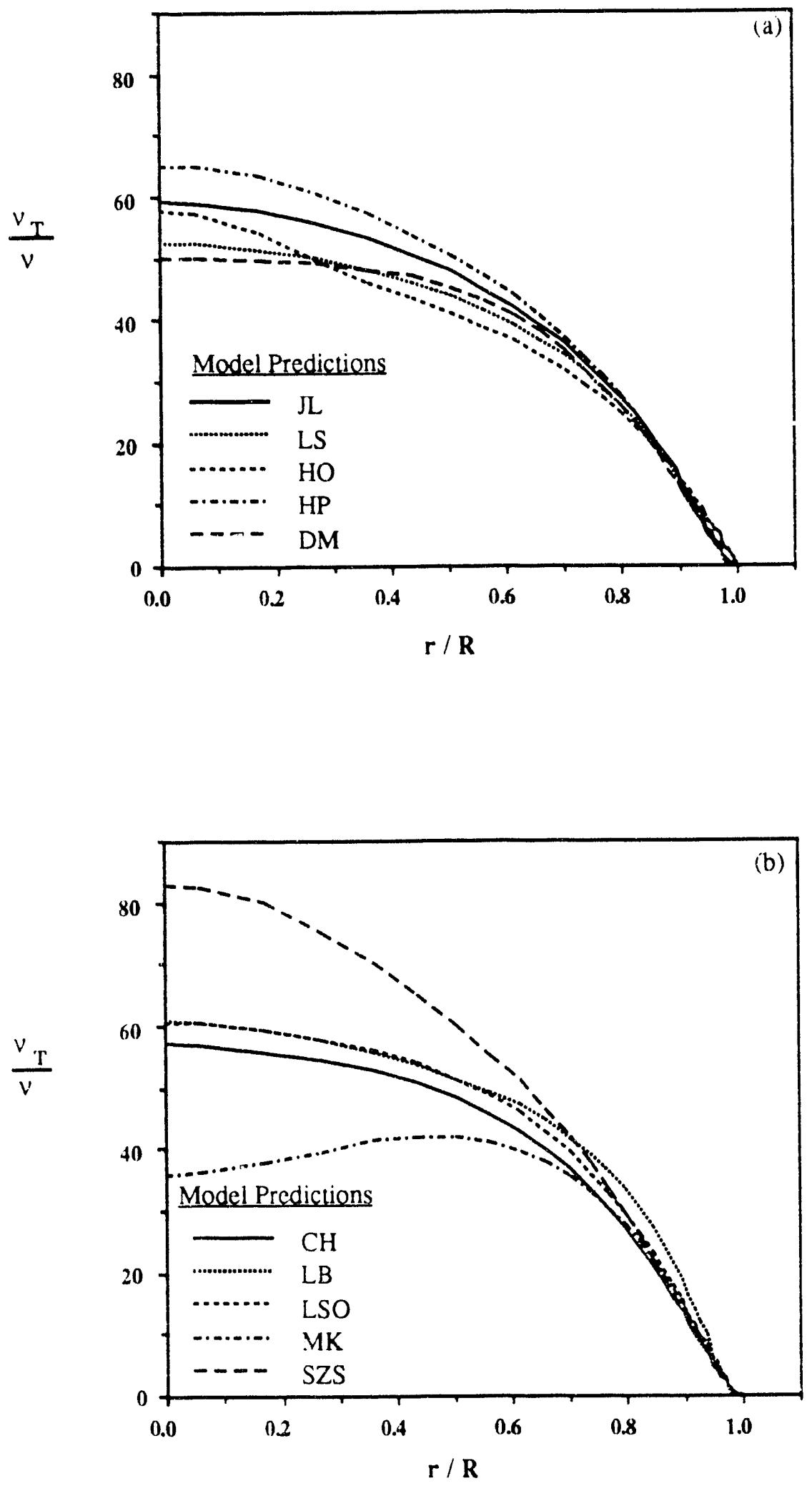

Figures 3.3a, 3.3b Model predictions for the eddy viscosity for the system of Schildknecht et al. 
In Figure 3.4, we show a plot of the prediction of the dimensionless dissipation rate $\varepsilon$ at the wall region. The model predictions for the MK and the SZS models are shown. An important qualitative difference is noticed. The MK $\varepsilon$ profile has a peak near the wall. The same behavior is predicted by all the other models, except the SZS model. The SZS model predicts a plateau where the other models reach the maximum value, but thereafter the dissipation rate increases reaching the maximum value at the wall. This behavior has been predicted by the direct numerical simulation of the Navier-Stokes equations.

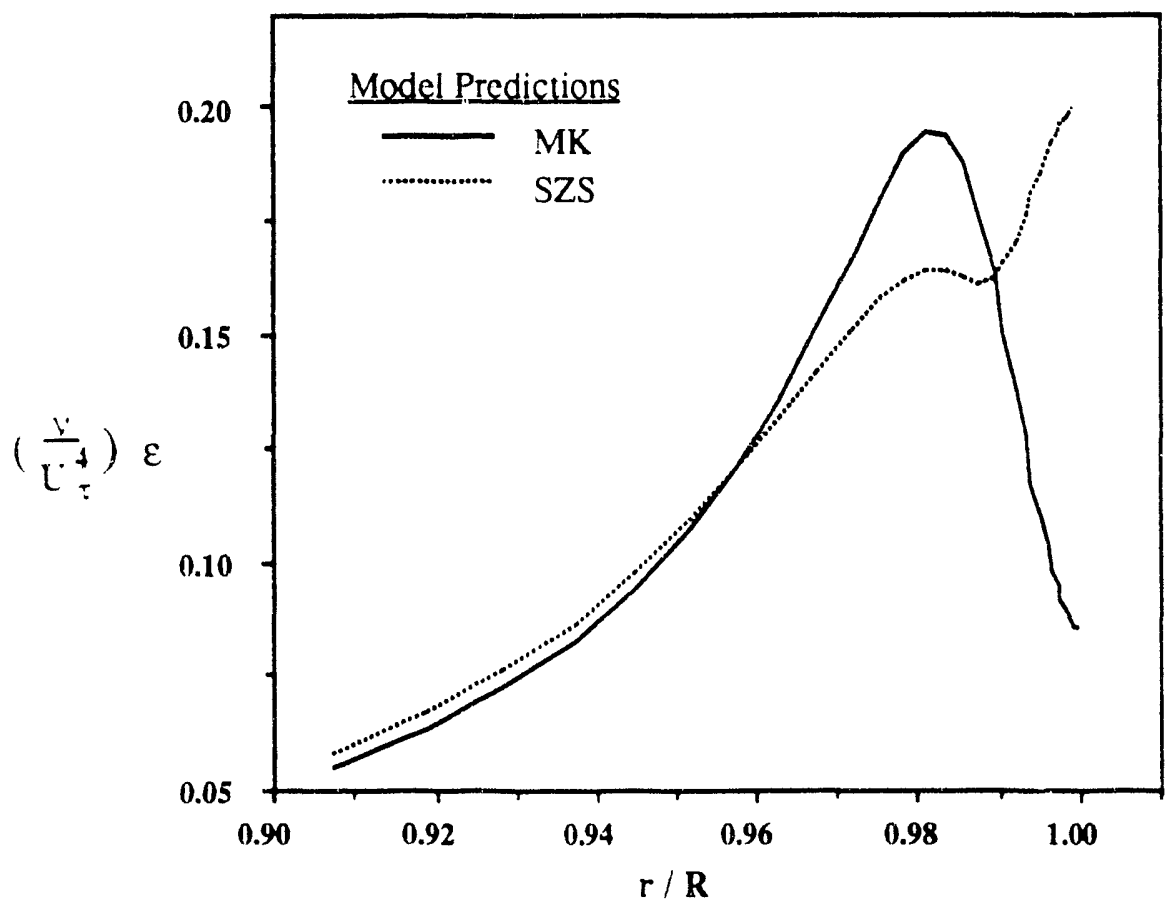

Figure 3.4 Model predictions for the turbulent kinetic energy dissipation rate in the wall region for the system of Schildknecht et al. The frictional velocity $U_{\tau}$ is $0.29 \mathrm{~m} / \mathrm{s}$. 


\section{Conclusions}

Before modifying a single-phase turbulence model to account for the presence of particles, we must be certain that the model can accurately predict single-phase pipe flow. From the comparisons of the ten low-Reynolds k- $\varepsilon$ closure models with the experimental measurements of Schildknecht $e t \omega$. , it is concluded that significant qualitative and quantitative differences exist among the closures. In order to further evaluate the ability of each of the models, comparisons of model predictions with additional experimental data which includes tur bulent kinetic energy and eddy viscosity profiles are needed. Specifically, the measurements to be considered include those of Laufer (1954) and Lawn (1971). 


\section{Sources Cited}

Dutoya. D. and P. Michard. 1981. A Program for Calculating Boundary Layers and Heat Transier along Compressor and Turbine Blades. In Numerical Methods in Heat Transfer. Edited by R. W. Lewis K. Morgan and O.C. Zienkiewicz. 413-428. John Wiley and Sons, Ltd.

Chien. K. 1982. Predictions of Channel and Boundary-Layer Flows with a LowReynoids-Number Turbulence Model. AIAA J. 20(1): 33-38.

Hassid. S. and M. Poreh. 1978. A. Turbulent Energy Dissipation Model for Flows With Drag Reduction. J. Fluid Eng. 100: 107-112.

Hoffman. G. H. 1975. Improved form of the low Reynolds number $k-\varepsilon$ turbulence model. Phys. Fluids 18(3): 309-312.

Jones. W. P., and B.E. Launder. 1972. The Prediction of Laminarization with a TwoEquation Model of Turbulence. Int. J. Heat Mass Transfer 15: 301-314.

Jones. W. P. and. B. E. Launder. 1973. The calculation of low-Reynolds-number phenomena with a two-equation model of turbulence. Int. J. Heat Mass's 'ransfer 16: 1119-1130.

Lai, Y.G. and R.M.C. So. 1990. On near-wall turbulent flow modelling. J. Fluid Mech. 221(641-673.

Lam. C.K.G. and K. Bremhorst. 1981. A Modified Form of the k- $\varepsilon$ Model for Predicting Wall Turbulence. J. Fluids Eng. 103(Sep. 1981): 456-460.

Laufer. John. 1954. The structure of turbulence in fully developed pipe flow. National Advisory Committee for Aeronautics (NACA). NTIS, 1174.

Launder. B. E. and. B. I. Sharma. 1974. Application of the Energy-Dissipation Model of Turbulence to the Calculation of Flow Near a Spinning Disc. Letters Heat Mass Transf. 1: $131-138$.

Martinuzzi, R. and. A. Pollard. 1989. Comparative Study of Turbulence Models in Predicting Turbulent Pipe Flow Part I: Algebraic Stress and k- $\varepsilon$ Models. AIAA J. 27(1): 29-36.

Myong. H.K. and N. Kasagi. 1990. A New Approach to the Improvement of k- $\varepsilon$ Turbulence Model for Wall-Bounded Shear Flows. JSME Int. J. Series II, 33(1): 63-72.

Patel. V., W. Ródi, and G. Scheuerer. 1985. Turbulence Models for Near-Wall and Low Reynoids Number Flows: A Review. AIAA J. 23(9): 1308-1319.

Pita. J. and. S. Sundaresan. 1991. Gas-Solid Flow in Vertical Tubes. AIChE J. 37(7): 1009-1018. 
Pollard, A. and. R. Martinuzzi. 1989. Comparative Study of Turbulence Models in Predicting Turbulent Pipe Flow Part II: Reynolds Stress and k-e Models. AIAA J. 27(12): $171+-1721$.

Rhodes, M.J. and D. Geldart. 1989. The Upward Flow of Gas/Solid Suspensions, Part 1: A Model for the Circulating Fluidized Bed Incorporating Dual Level Gas Entry into the Raiser. Chem. Eng. Res. Des. 67: 20-37

Rodi. W. 1982. Examples of Turbulence Models for Incompressible Flows. AIAA J. 20(7): 872-879.

Schildknecht, M., Miller, J.A. and G.E. Meier. 1979. The influence of suction on the structure of turbulence in fully developed pipe flow. J. Fluid Mech. 90, part I: 67-107.

Shiir. T. I. 1988. Mathematical Models for the Numerical Study of Turbulent Flows. $J$. Chinese Inst. Eng. 11(2): 121-136.

So. R. M. C., H.S. Zhang and C.G. Speziale. 1991. Near-Wall Modeling of the Dissipation Rate Equation. AIAA J. 29(12): 2069-76. 

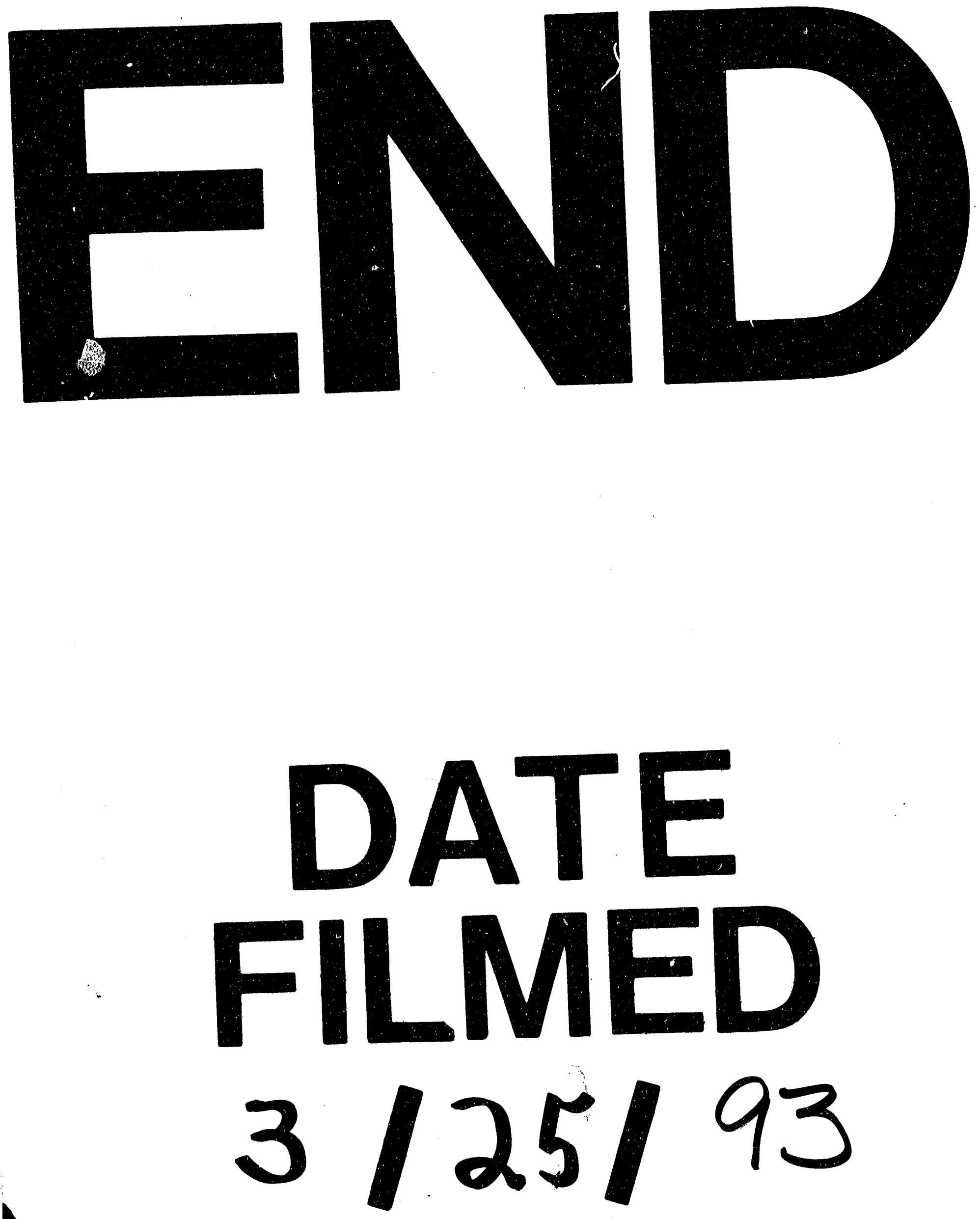

1 
